\title{
The magnetic reversal in dot arrays recognized by the self-organized adaptive neural network
}

\author{
Martin Gmitra and Denis Horváth \\ Department of Theoretical Physics and Astrophysics, \\ University of P.J.Šafárik, \\ Moyzesova 16, 04001 Košice, \\ Slovak Republic
}

\begin{abstract}
The remagnetization dynamics of monolayer dot array superlattice XY 2-D spin model with dipole-dipole interactions is simulated. Within the proposed model of array, the square dots are described by the spatially modulated exchange-couplings. The dipole-dipole interactions are approximated by the hierarchical sums and spin dynamics is considered in regime of the LandauLifshitz equation. The simulation of reversal for 40000 spins exhibits formation of nonuniform intra-dot configurations with nonlinear wave/anti-wave pairs developed at intra-dot and inter-dot scales. Several geometric and parametric dependences are calculated and compared with oversimplified four-spin model of reversal. The role of initial conditions and the occurrence of coherent rotation mode is also investigated. The emphasis is on the classification of intra-dot or interdot (interfacial) magnetic configurations done by adaptive neural network with varying number of neurons.
\end{abstract}

PACS: Nanostructures: 79.60.J, Magnetostatics 41.20.G, Magnetic ordering 75.10, Numerical simulation studies $75.40 . \mathrm{Mg}$

Key words: magnetic dot array, neural network model, XY model, numerical simulation

\section{Introduction}

Many interesting applications of the nanometer periodic magnetic dot arrays are expected in the magnetic recording [1] and magnetic sensors. The magnetic nanostructures represent model systems for study of interesting physical phenomena stemming from the well controlled dot shape anisotropy and dominance of magnetostatic interactions at the scales comparable or larger than dot size. The magnetostatics cause the coupling of domains over the inter-dot interfaces. The current perspective technologies require understanding and controlling of dynamic magnetization processes in fine particles on nanoscales, because by them the magnetic bits are written into recording media. Hence of particular interest is the dynamics of magnetization reversal.

The static and dynamic nonuniform magnetization states in magnetic nanostructures including the thin films and isolated small particles have been studied by micromagnetic $[2,3,4]$ and Monte Carlo $[5,6,7,8]$ simulations. On a qualitative level intra-dot nonuniformities can be characterized as a superpositions of vortices, flowers, domain walls, etc.. The problem of nonuniformities in exchangedominated structures can be solved exactly [9].

Our recent activity is directed to algorithms allowing systematic and fast classification of simulated magnetic structures. The methodology presented in this paper is inspired by the progress in the theory of artificial neural networks that are nonlinear models suitable to reduce, classify or interpolate data structures representing the complex patterns [10]. In the previous paper [11] we suggested the implementation of radial basis function networks to model the ordering of dots in arrays. In the next paper [12] the neural network training was applied to multiscale numerical computations. The lack of our previous two approaches was the absence of realistic dynamics and restriction to quasi-static limit. In the present paper our interest is focused to the networks which analyze spin configurations 
generated by the spin lattice dynamical simulations. It means that network solves here classical postprocessing problem known in the micromagnetic context [13]. Our approach has many in common with experiment treatment [14], where self-organized network was employed to identification of vortex modes of magnetic thin film media. In the presented paper the classification is applied to configurations generated by the magnetization reversal within the XY superlattice model of ultra-dense array. The motivation for study of ultra-dense spin structures was the expectation of extremal inter-dot effects.

The main reason for the choice of the XY spin model is relative computational simplicity of 2-D dynamics. But there are two regimes for which 2-D approximation is justified physically. (i) The first regime belongs to limit of very strong damping which suppresses precession and accelerates reversal [15]. (ii) The second regime represents limit of strong in-plain [16] or shape anisotropy associated with demagnetization field of thin film sample [17]. In this regime anisotropy causes deformation of spin trajectory and accelerates reversal [18]. Nevertheless, one should to note that realistic damping is sufficiently small, and thus $2-\mathrm{D}$ remagnetization is practically quasistatic. In the quasistatic limit the damping parameter has meaning of auxiliary parameter rescaling the time. Recent results obtained for discrete spin systems provide more optimistic view on the valuation of artificial dynamics of reversal. The example is the simulation of [19] demonstrating that switching time Monte Carlo dependences can coincide after some rescaling with predictions of the stochastic Landau-Lifshitz equation.

The plan of the paper is the following. The interaction picture and dynamics of array model are defined in section 2. The simulations of array reversal are presented and discussed in section 3. The details of adaptive neural network treatment of intra-dot configurations are given in subsection 3.3.

\section{XY periodically modulated superlattice model of array}

In this section we formulate the interaction picture and dynamics of ultra dense array model. The magnetic ordering is described by the systems of the classical planar spins located on the square two dimensional lattice. The spin on site $\alpha$ is affected by effective field

$$
\mathbf{h}_{\alpha}^{\mathrm{eff}}=\frac{D}{J} \sum_{\substack{\beta=0 \\ \beta \neq \alpha}}^{L^{2}-1} \frac{3 \mathbf{n}_{\alpha \beta}\left(\mathbf{n}_{\alpha \beta} \cdot \mathbf{S}_{\beta}\right)-\mathbf{S}_{\beta}}{\left|\mathbf{r}_{\alpha \beta}\right|^{3}}+\sum_{\substack{\beta=0 \\\left|\mathbf{r}_{\alpha \beta}\right|=1}}^{L^{2}-1} \epsilon_{\alpha \beta} \mathbf{S}_{\beta}+\frac{\mathbf{H}}{J},
$$

where $\mathbf{S}_{\alpha}=S_{\alpha \mathrm{x}} \mathbf{e}_{\mathrm{x}}+S_{\alpha \mathrm{y}} \mathbf{e}_{\mathrm{y}}$ is the planar spin, $\mathbf{H}$ is the external magnetic field in energy units, $L$ is the linear size of the square $L \times L$ spin lattice system. Here and in further $\mathbf{e}_{\mathrm{x}}, \mathbf{e}_{\mathrm{y}}$ denote the unit vectors along the main Cartesian axes. Rather convenient for a computer purposes is to use one dimensional enumeration scheme of spin sites $\alpha \in\left\{0,1,2, \ldots L^{2}-1\right\}$. (All of the geometric details presented in this section are discussed in the Appendix A.1.) The first sum of Eq.(1) represents dipolar contribution proportional to constant $D$, where $\mathbf{r}_{\alpha \beta}$ is vector pointing from site $\alpha$ to $\beta$. The vector is measured in lattice constant units, $\mathbf{n}_{\alpha \beta}=\mathbf{r}_{\alpha \beta} /\left|\mathbf{r}_{\alpha \beta}\right|$ is the auxiliary unit vector. The second sum of Eq.(1) involves nearest neighbor exchange coupling term $J \epsilon_{\alpha \beta}$ determined by single parameter $J>0$ multiplying the site-site connectivity matrix $\epsilon_{\alpha \beta} \in\{1,0\}$ constrained by $\left|\mathbf{r}_{\alpha \beta}\right|=1$ condition; $\epsilon_{\alpha \beta}$ is 0 for any bond at inter-dot boundary and 1 inside a dot. The site dependence of $\epsilon_{\alpha \beta}$ takes into account square shape of dot of the linear size $L_{0}$. The spin system partitioned by $\epsilon_{\alpha \beta}$ consists of $L_{\mathrm{d}}^{2}=\left(L / L_{0}\right)^{2}$ dots with minimum inter-dot distance equal to the unit lattice spacing and corresponding to extremal space filling.

In 2-D case Landau-Lifshitz equation reduces to damping term. The numerical integration scheme discussed in Appendix A.2 can be derived from recursive propagator form

$$
\mathbf{S}_{\alpha}(\tau+\Delta \tau)=\hat{\mathbf{U}}\left(\int_{\tau}^{\tau+\Delta \tau} \omega_{\alpha}\left(\tau^{\prime}\right) \mathrm{d} \tau^{\prime}\right) \mathbf{S}_{\alpha}(\tau)
$$

where $\omega_{\alpha}(\tau)=\left(\mathbf{S}_{\alpha}(\tau) \cdot \mathbf{e}_{\mathrm{x}}\right)\left(\mathbf{h}_{\alpha}^{\mathrm{eff}}(\tau) \cdot \mathbf{e}_{\mathrm{y}}\right)-\left(\mathbf{S}_{\alpha}(\tau) \cdot \mathbf{e}_{\mathrm{y}}\right)\left(\mathbf{h}_{\alpha}^{\mathrm{eff}}(\tau) \cdot \mathbf{e}_{\mathrm{x}}\right)$ is instant angular frequency of the spin rotation, $\Delta \tau$ is the time integration step, $\tau$ is the reduced time related to real time $t$ as $\tau=\mu_{0} \gamma M_{\mathrm{s}} t$, where $\mu_{0}$ is permeability of free space, $\gamma$ is the gyromagnetic ratio and $M_{\mathrm{S}}$ is the saturation magnetization. The unitarity of spin rotation matrix

$$
\hat{\mathbf{U}}(\varphi)=\left(\begin{array}{rr}
\cos \varphi & -\sin \varphi \\
\sin \varphi & \cos \varphi
\end{array}\right)
$$


implies that numerical scheme adapted from Eq.(2) conserves the spin vector size independently of $\int_{\tau}^{\tau+\Delta \tau} \omega_{\alpha}\left(\tau^{\prime}\right) \mathrm{d} \tau^{\prime}$ quadrature.

The time demanding task represents summation of dipolar fields. The substantial reduction of time allows the use of the hierarchical approach [20] with a detailed summation of near and rough summation of far field contributions. The specific geometry of array leads to the natural spin block definition

$$
\mathbf{S}_{j}^{\mathrm{d}}=\sum_{\substack{\beta=0 \\ i(\beta)=j}}^{L^{2}-1} \mathbf{S}_{\beta}, \quad j \in\left\{0,1,2, \ldots, L_{\mathrm{d}}^{2}-1\right\},
$$

where sum runs over the spins of index $\beta$ belonging to dot index $i(\beta)=j$ and dot position $\mathbf{r}_{i(\beta)}^{\mathrm{d}}$. In frame of two-level hierarchical approximation dipolar field is splitted to near $\mathbf{h}_{\alpha}^{\text {eff,N }}$ and far $\mathbf{h}_{\alpha}^{\text {eff, } F}$ field

$$
\begin{aligned}
& \mathbf{h}_{\alpha}^{\mathrm{eff}, \mathrm{N}}=\frac{D}{J} \sum_{\substack{\beta=0, \beta \neq \alpha \\
\left|\mathbf{r}_{i(\alpha), i(\beta)}^{\mathrm{d}}\right| \leq r_{\text {cut }}}}^{L^{2}-1} \frac{3 \mathbf{n}_{\alpha \beta}\left(\mathbf{n}_{\alpha \beta} \cdot \mathbf{S}_{\beta}\right)-\mathbf{S}_{\beta}}{\left|\mathbf{r}_{\alpha \beta}\right|^{3}}+\sum_{\substack{\beta=0 \\
\left|\mathbf{r}_{\alpha \beta}\right|=1}}^{L^{2}-1} \epsilon_{\alpha \beta} \mathbf{S}_{\beta}+\frac{\mathbf{H}}{J} \\
& \mathbf{h}_{\alpha}^{\mathrm{eff}, \mathrm{F}}=\frac{D}{J} \sum_{\substack{j=0 \\
\left|\mathbf{r}_{i(\alpha) j}^{\mathrm{d}}\right|>r_{\text {cut }}}}^{L_{\mathrm{d}}^{2}-1} \frac{3 \mathbf{n}_{i(\alpha) j}^{\mathrm{d}}\left(\mathbf{n}_{i(\alpha) j}^{\mathrm{d}} \cdot \mathbf{S}_{j}^{\mathrm{d}}\right)-\mathbf{S}_{j}^{\mathrm{d}}}{\left|\mathbf{r}_{i(\alpha) j}^{\mathrm{d}}\right|^{3}},
\end{aligned}
$$

depending on the unit vectors $\mathbf{n}_{i(\alpha) j}^{\mathrm{d}}=\mathbf{r}_{i(\alpha) j}^{\mathrm{d}} /\left|\mathbf{r}_{i(\alpha) j}^{\mathrm{d}}\right|, \mathbf{r}_{i(\alpha) j}^{\mathrm{d}}=\mathbf{r}_{j}^{\mathrm{d}}-\mathbf{r}_{i(\alpha)}^{\mathrm{d}}$. The summation rule is controlled by the scale-separation cut-off $r_{\text {cut }}$.

\section{The results of array reversal simulations}

In this section we present the simulations of the square shaped finite array including 40000 spins. To analyze the simulation outputs we applied: (i) the qualitative recognition of the configuration snapshots; (ii) the comparing of the complex array dynamics with the dynamics of much simpler systems; (iii) the clustering of intra-dot magnetic configurations using neural networks. The global information on the array ordering concerns the magnetization per site

$$
\mathbf{M}(\tau)=\frac{1}{L^{2}} \sum_{\alpha=0}^{L^{2}-1} \mathbf{S}_{\alpha}(\tau)
$$

The simulations were performed for uniform zero $\left.\mathbf{S}_{\alpha}\right|_{\tau=0}=-\mathbf{H} / H=\mathbf{e}_{\mathrm{x}}$ and non-zero bias $\left.\mathbf{S}_{\alpha}\right|_{\tau=0}=$ $\cos \left(\varphi_{0}\right) \mathbf{e}_{\mathrm{x}}+\sin \left(\varphi_{0}\right) \mathbf{e}_{\mathrm{y}}, \varphi_{0} \neq 0$ initial conditions. In both cases the characteristic time of array is the time when $\mathbf{H} \cdot \mathbf{M}$ projection gains at first the bound

$$
\mathbf{H} \cdot \mathbf{M}\left(\tau_{\mathrm{ch}}\right)=0 .
$$

\subsection{The qualitative approach based on two-scale similarity}

To obtain the preliminary qualitative information about the switching mechanism we run the simulation of reversal dynamics in the field $H / J=-1$ and coupling $D / J=0.1$ for dot size $L_{0}=20$ and bias $\varphi_{0}=0$. Under these conditions we obtained estimate $\tau_{\text {ch }}=8.516$.

At the beginning of reversal the external magnetic field nucleates reversal domains at the corners of dots. The recognition of simulated systems shows that remagnetization is more homogeneous and easier when dots are localized at the corners of array, whereas nonuniformities occur due to mirror symmetry of effective fields typical for dots standing in positions closer to axial zone of array. This property is illustrated by snapshot taken at characteristic time as is shown in Fig. 1. The typical for nonuniform remagnetization is that reverse domains, which gain external field polarity, grow from the dot corners and pass toward the dot centers. At the intermediate stages domain walls - nonlinear waves [5] between reverse and initial polarity domains become narrow. In the context with nanoparticles 
and our small-scale simulations we prefer to use the term nonlinear wave instead of domain wall since nonlinear wave better expresses nonlocal character of arising spin structures. At the final stage of the inhomogeneous reversal the sum of external and dipolar field can, if sufficiently strong, homogenize intra-dot ordering by annihilating of wave/anti-wave pairs (WAP). Similar mechanism was studied in detail for linear spin chain [21]. As we see from the scheme depicted in Fig. 2 the reversal mechanism in dots is position and time dependent for finite size arrays. The formation of WAP is an example of inhomogeneous remagnetization. The process is characteristic for later times and dots placed nearly the axial array zone as is depicted in Fig. 3. The parallelism between spin chain and array behavior inspired the monitoring of spin projections summarized in Fig. 4. The figure shows $S_{\alpha y}$ components taken from several superlattice site lines crossing array in external field direction. The time sequence of these projections reveals that each inter-dot interface acts as a phase-shifter of WAP formed in different dots. The consequence of inter-dot coupling is the large-scale WAP with shape resembling its smallscale intra-dot counterpart (see Fig. 5). It is rather surprising that for its build up magnetostatic forces suffice. The spatio-temporal two-scale similarity of magnetic ordering illustrated in Fig. 6 originates from spatial distribution of dipolar fields of square-shaped dots embedded into square-shaped array.

\subsection{The quantitative analysis of switching, the effect of bias}

\subsubsection{The dot-size dependences}

We continue with the comparing of $\tau_{\mathrm{ch}}\left(L_{0}\right)$ dependences for patterned structure as well as for the isolated dots with zero and non-zero bias. The varying of $L_{0}$ is in some sense equivalent to varying of dipolar and exchange coupling contributions. The results for patterning $L_{0}=5,10,20,25,40,50$ for $D / J=1$ are depicted in Fig. 7, where the characteristic times of the interacting and isolated dots are also compared. From the simulations it follows that inhomogeneities grow as $L_{0}$ increases. The reverse phase nucleates preferentially at dot surfaces and reduces $\tau_{\mathrm{ch}}$ in larger dots. If inter-dot interactions are considered $\tau_{\mathrm{ch}}\left(L_{0}\right)$ dependences look smoother than dependences obtained for isolated dots. The interactions affect spatial dispersion of $\omega_{\alpha}$, which means that some dots are accelerated and some decelerated during reversal. The simulations of isolated dots show that differences between zero and non-zero bias are larger for smaller $L_{0}$. The decrease of $\tau_{\text {ch }}$ due to uniform bias $\varphi_{0}>0$ is a manifestation of the Stoner-Wohlfarth coherent regime.

\subsubsection{The interplay of $D, J, H, \varphi_{0}$ parameters, minimal four-spin model}

In this section minimal four-spin model and complete array model of magnetization reversal are discussed and compared. The minimal model consists of four spins arranged to $2 \times 2$ square segment of unit size. In the segment four nearest pairs are coupled by the exchange interaction and all of the pairs by dipole-dipole interaction. Despite of substantial geometric truncation, all of the interactions considered for array take place in four-spin model. The simulation of the minimal model system provides $\tau_{\text {ch }}^{\mathrm{f}}$ characteristics depicted in Fig. 8. They consist of two qualitatively different regimes separated by bifurcation point $D=D_{\mathrm{c}}^{\mathrm{f}}$ manifested by $\tau_{\mathrm{ch}}^{\mathrm{f}}(D)$ cusp. At $D_{\mathrm{c}}^{\mathrm{f}}$ several modes compete, the interaction and external fields are nearly balanced and frustrated spins need quite long time to select the winning mode. For $D<D_{\mathrm{c}}^{\mathrm{f}}$ the reversal is very susceptible to choice of initial bias. It is easy to check that even small $\varphi_{0}>0$ accelerates reversal and stabilizes coherent or quasi-coherent rotation in coincidence with the Stoner-Wohlfarth scenario. Further simplification of four-spin dynamics which clarifies situation can be done assuming the total coherence of spins. The consequence is the elimination of three angular variables and analytic expression

$$
\tau_{\mathrm{ch}}^{\mathrm{f}}=\frac{4 \sqrt{2} J}{\mathcal{L}} \int_{\varphi_{0}}^{\pi / 2} \frac{\mathrm{d} \varphi}{3 D \cos (2 \varphi)+4 \sqrt{2} H \sin \varphi} .
$$

where $\mathcal{L}=4 \pi \lambda / \gamma \mu_{0} M_{\mathrm{s}}$ is undimensional parameter proportional to damping parameter $\lambda$. The expression obtained in the limit $D \rightarrow 0$ is rather well known

$$
\tau_{\mathrm{ch}}=\frac{J}{H \mathcal{L}} \int_{\varphi_{0}}^{\pi / 2} \frac{\mathrm{d} \varphi}{\sin \varphi}=\frac{J}{H \mathcal{L}} \ln \left(\operatorname{cotg}\left(\varphi_{0} / 2\right)\right) .
$$

For $D>0$ the integral Eq.(9) leads to finite $\tau_{\text {ch }}^{\mathrm{f}}$ even in the case $\varphi_{0}=0$. It means that dipolar coupling removes logarithmic divergence $\propto \ln \varphi_{0}$ of $\tau_{\text {ch }}^{\mathrm{f}}$ and substitutes in part the effect of $\varphi_{0}>0$. 
In the regime $D<D_{\mathrm{c}}^{\mathrm{f}}$ the bias $\varphi_{0}=0$ induces non-uniform flower mode belonging to characteristic time which is out of the scope of Eq.(9). The four-spin dynamics attained by simulation for $D>D_{\mathrm{c}}^{\mathrm{f}}$ is even more complex. In that case the choice of bias losses its dynamical relevance. The simulations show that quite short time is needed to form the transient long-living flower mode (long with respect to $\tau_{\mathrm{ch}}^{\mathrm{f}}$ ) which alters to vortex mode at final stages.

With the four-spin system behavior in mind the attention is turned now to the complex array dynamics. As we see from Fig. $9 \tau_{\mathrm{ch}}(D / J)$ dependence does not contain sharp cusp. Instead of it, the slope variation of $\tau_{\mathrm{ch}}(D / J)$ arises (labeled as $D_{\mathrm{c}}$ in analogy to $D_{\mathrm{c}}^{\mathrm{f}}$ ). For $L_{0}=20$ and $H / J=1$ the position of $D_{\mathrm{c}}$ can be estimated as $D_{\mathrm{c}} \simeq 5 \mathrm{~J}$. The configuration snapshots show that near $D_{\mathrm{c}}$ the array consists of spin configuration loops of diameters approaching single dot size or size of several dots. For $D>D_{\text {c }}$ the array reversal generates non-collinear antiferromagnetic phases. The common feature of oversimplified four-spin model and array simulation is that finite $\tau_{\text {ch }}^{\mathrm{f}}$ and $\tau_{\text {ch }}$ are attained even for $\varphi_{0}=0$ due to non-collinearity of external and dipolar fields. From dependences depicted in Figs. 7 and 9 it follows that geometric variations in $L_{0}$ can produce less remarkable changes than $D / J$ parameters.

For completeness the external field dependences of characteristic time should be mentioned. From Eq.(9) one can obtain for $D / H \ll 1$ the asymptotic expansion of characteristic time $\tau_{\text {ch }}^{\mathrm{f}}=(J /(\mathcal{L} H))$ $\sum_{n=0}^{\infty}(-1)^{n}(D / H)^{n} c_{n}\left(\varphi_{0}\right), c_{n}\left(\varphi_{0}\right)=3^{n} 2^{-5 n / 2} \int_{\varphi_{0}}^{\pi / 2} \mathrm{~d} \varphi \cos ^{n}(2 \varphi)(\sin (\varphi))^{-n-1}$ suitable for proposition of reasonable fitting function covering the array data. The array simulations in Fig. 10 shows that $\tau_{\mathrm{ch}}$ coincides qualitatively with asymptotic predictions of four-spin system.

\subsection{The intra-dot complex structures in representation of ART network}

The artificial neural network models [10] inspired by the physiology are able to mimic action of neurons and synaptic connections of brain. The salient feature of neural systems is the associative recognition of the complex structures. This ability is attained by training of synaptic connections - neurons. In this paper we deal with networks based on the adaptive resonance theory (ART) developed by Carpenter and Grossberg [22] originally as a model explaining adaptive phenomena in visual systems. The family of ART algorithms belongs to unsupervised self-organized algorithms motivated by need to construct network sufficiently adaptive to novel inputs. The adaptivity means that network produces new neurons if diversity of inputs overcomes the predefined threshold represented by so called vigilance parameter. On the other hand redundancy of information leads to the annihilation of neurons. For summary of ART network applications see e.g. [23]

\subsubsection{The encoding of intra-dot magnetic structure}

The ideas of clustering and adaptivity of the ART networks led us to their application to magnetic intra-dot configurations generated during reversal. At first, an appropriate format for encoding of magnetic configurations is suggested. Similarly to formulation [11] the configurations are encoded by $2 N_{\mathrm{c}}$ dimensional tuples

$$
\tilde{m}_{i} \equiv\left[\mathbf{m}_{i 1}, \mathbf{m}_{i 2}, \ldots, \mathbf{m}_{i N_{\mathrm{c}}}\right],
$$

where

$$
\mathbf{m}_{\text {in }}=\frac{1}{N_{\mathrm{S}}} \sum_{l \in \square_{i n}} \mathbf{S}_{l}, \quad i=1,2, \ldots, L_{\mathrm{d}}^{2}, \quad n=1,2, \ldots, N_{\mathrm{c}}
$$

is the local average taken from $N_{\mathrm{S}}$ spins (see Fig. 11), where sum runs over site $l$ taken from $n$th segment of $i$ th dot labeled as $\square_{i n}$. Each dot is subdivided to $N_{\mathrm{c}}=L_{0}^{2} / N_{\mathrm{S}}$ intra-dot square segments. The system of $L_{\mathrm{d}}^{2}$ intra-dot configurations is transformed into $\tilde{m}_{i}$ tuples incoming to ART, which compresses them to $N_{\mathrm{w}} \leq L_{\mathrm{d}}^{2}$ neurons

$$
\tilde{w}_{j} \equiv\left[\mathbf{w}_{j 1}, \mathbf{w}_{j 2}, \ldots, \mathbf{w}_{j N_{\mathrm{c}}}\right], \quad j \in\left\{1,2, \ldots N_{\mathbf{w}}\right\}
$$

representing the basal types of intra-dot ordering. The self-organization mechanism of neurons discussed in the next is controlled by the coincidence between input $\tilde{m}_{i}$ and output $\tilde{w}_{j}$. As a proper measure of coincidence is chosen Euclidean distance

$$
\left\|\tilde{w}_{j}-\tilde{m}_{i}\right\|=\sqrt{\sum_{n=1}^{N_{\mathrm{c}}}\left(\mathbf{w}_{j n}-\mathbf{m}_{i n}\right) \cdot\left(\mathbf{w}_{j n}-\mathbf{m}_{i n}\right)^{\mathrm{T}}},
$$


where T labels transposition.

\subsubsection{The ART clustering algorithm}

The training algorithm is described in the following points

1. Initialization setting $N_{\mathrm{w}}=1, \tilde{w}_{1}=\tilde{m}_{i}$ for random dot selection between $i \in\left\{0,1, \ldots, L_{\mathrm{d}}^{2}-1\right\}$.

2. Loop through the set of intra-dot magnetic configurations. For each randomly selected dot $i$ follows:

2.1 Presentation of $\tilde{m}_{i}$ to ART network. The training is realized for $k=1,2, \ldots$ iteration steps.

2.2 Computing of the actual index $j^{*}(k, i)$ of the fired neuron $\tilde{w}_{j^{*}(k, i)}(k)$ trained by $\tilde{m}_{i}$ pattern according to the competitive rule

$$
j^{*}(k, i)=\arg \min _{j=1,2, \ldots, N_{\mathrm{w}}}\left\|\tilde{w}_{j}(k)-\tilde{m}_{i}\right\| .
$$

2.3 Comparing $\left\|\tilde{w}_{j^{*}(k, i)}(k)-\tilde{m}_{i}\right\|$ to the vigilance parameter $\rho$.

2.3.1 Update of the neuron weights via Hebbian training rule [10]

$$
\tilde{w}_{j^{*}(k, i)}(k+1)=\tilde{w}_{j^{*}(k, i)}(k)+\eta(k)\left(\tilde{m}_{i}-\tilde{w}_{j^{*}(k, i)}(k)\right)
$$

is applied if $\left\|\tilde{w}_{j^{*}(k, i)}-\tilde{m}_{i}\right\| \leq \rho$. The update shifts winning vector toward nearby input with rate proportional to plasticity parameter $\eta(k)=\eta_{0} \exp \left(-k / \tau_{\text {train }}\right)$ relaxing with training time $\tau_{\text {train }}$.

2.3.2 Creation of new neuron $N_{\mathrm{w}} \leftarrow N_{\mathrm{w}}+1, \tilde{w}_{N_{\mathrm{w}}} \leftarrow \tilde{m}_{i}$ if $\left\|\tilde{w}_{j^{*}(k, i)}(k)-\tilde{m}_{i}\right\|>\rho$.

2.4 Annihilation of neuron pair $\left[\tilde{w}_{z_{1}^{*}}(k), \tilde{w}_{z_{2}^{*}}(k)\right]$, ordered as $z_{1}^{*}<z_{2}^{*}$, with indices $z_{1}^{*}$, $z_{2}^{*}$ selected according to minimum distance

$$
\left[z_{1}^{*}, z_{2}^{*}\right]=\arg \min _{z_{1}, z_{2}}\left\|\tilde{w}_{z_{1}}-\tilde{w}_{z_{2}}\right\|
$$

if $\left\|\tilde{w}_{z_{1}^{*}}-\tilde{w}_{z_{2}^{*}}\right\|<\rho$. The product of annihilation $\left[z_{1}^{*}, z_{2}^{*}\right] \rightarrow z_{1}^{*}, N_{\mathrm{w}} \leftarrow N_{\mathrm{w}}-1$ is the neuron $\tilde{w}_{z_{1}^{*}}$ determined by the midpoint rule $\tilde{w}_{z_{1}^{*}} \leftarrow \frac{1}{2}\left(\tilde{w}_{z_{1}^{*}}+\tilde{w}_{z_{2}^{*}}\right)$. After this update of $\tilde{w}_{z_{1}^{*}}$ the neurons having $j \geq z_{2}^{*}$ are rewritten: $\tilde{w}_{j-1} \leftarrow \tilde{w}_{j}$.

3. Stop criterion is represented by the inequality

$$
\frac{1}{L_{\mathrm{d}}^{2}} \sum_{i=0}^{L_{\mathrm{d}}^{2}-1}\left\|\tilde{w}_{j^{*}(k, i)}(k+1)-\tilde{w}_{j^{*}(k, i)}(k)\right\|<\varepsilon,
$$

where $\varepsilon$ is small parameter. If the above inequality is not fulfilled, the algorithm follows from the step 2 with $k$ incremented by 1 .

The stop criterion indicates when the network attains a fixed point of $\tilde{w}_{j}, j=1,2, \ldots, N_{\mathrm{w}}$ including the cluster of typical representative intra-dot configurations.

\subsubsection{The ART applied to different stages of reversal}

The ART network has been applied independently to array configurations attained at selected stages of reversal. The results of self-organization are presented in Figs. 13-15. The ART self-organization was carried out for tuned parameters $\eta_{0}=0.1, \tau_{\text {train }}=20, \varepsilon=10^{-6}, \rho=1.15$. The most important seems to be optimized choice of an appropriate vigilance connecting $\rho$ and $N_{\mathrm{w}}$. It stem from calibration in Fig. 12. During it $\rho$ was decreased slowly from the initial $\rho=1.5$ stabilizing single neuron. This decrease of $\rho$ was stopped for $N_{\mathrm{w}}=L_{\mathrm{d}}^{2}=100$ competing neurons.

The ART view point on reversal leads to intra-dot taxonomy depicted in Fig. 13. In this figure the most similar neurons [in the sense of generalized distance Eq.(14)] at different times are joined by the arrows. The ART model clearly distinguishes between branches of coherently rotating monodomain dots and dots with more complex order. The diversity of configurations occurring near the internal 
energy maxima is reflected by the cardinality of neuron population. The ART pruning at late time stages is associated with small intra-dot diversity within the array. How choice of $\rho$ affects the information content of network is clearly demonstrated in Fig. 14. The supplementary problem solved by ART represents classification of interfacial inter-dot configurations. In that case the configuration tuples $\tilde{m}_{i}, \tilde{w}_{j}$ have been combined from $\mathbf{m}_{i n}$ segments [see Eq.(12)] of adjacent quadrants of four neighboring dots. The results depicted in Fig. 15 indicate preferential formation of inter-dot vortices and strong tendency to inter-dot pinning also for conditions when formation of intra-dot vortices is disadvantageous.

\section{Conclusions}

The magnetization reversal has been simulated for superlattice spin model of the ultra-dense magnetic dot array. Several aspects of reversal dynamics for different exchange and magnetostatic couplings, external fields, geometric parameters and initial conditions have been studied. It was found that the principal physical consequence of geometric modulation is the formation of two-scale WAP. The ART viewpoint seems to be fruitful in thinking how dot switches from one configuration to another within the large time intervals, and how to identify dominant channels of intra-dot evolution. The present experience creates believe that advantages of ART paradigm should be exploited namely in 3-D lattices, where visual classification meets natural bounds.

\section{Acknowledgments}

The work was partially supported by the grant No.1/6020/99 (Slovak Grant Agency VEGA). M. Gmitra expresses thanks to Dr. Z. Frait and Dr. V. Kamberský from Institute of Physics AS CR, Prague, for their hospitality and valuable discussions during stay supported by training network contract HPRN-CT-1999-00150.

\section{A Appendix}

\section{A.1 One dimensional enumeration scheme of superlattice sites}

In the Appendix site enumeration scheme mentioned in section 2 is described. In this scheme the superlattice relative position vector $\mathbf{r}_{\alpha \beta}$ pointing from site $\alpha$ to site $\beta$ is equal to

$$
\mathbf{r}_{\alpha \beta}=[\mathcal{M}(\beta, L)-\mathcal{M}(\alpha, L)] \mathbf{e}_{\mathbf{x}}+[\mathcal{I}(\beta, L)-\mathcal{I}(\alpha, L)] \mathbf{e}_{\mathrm{y}},
$$

where

$$
\mathcal{M}(a, b)=a \bmod b, \quad \mathcal{I}(a, b)=[a / b]_{\mathrm{int}} .
$$

Here $\mathcal{M}$ is standard modulo function and $\mathcal{I}$ is the integer part of its argument $(L$ is an integer multiple of $L_{0}$ ). The second part of Eq.(1) includes the exchange coupling $J \epsilon_{\alpha \beta}$ generated by dependence

$$
\epsilon_{\alpha \beta}=\left(1-\delta_{\phi(\beta)-1, \phi(\alpha)-L_{0}}\right)\left(1-\delta_{\xi(\beta)-1, \xi(\alpha)-L_{0}}\right) .
$$

It gives rise to geometry of dense square patterning of the period $L_{0}$ in perpendicular $\mathbf{e}_{\mathrm{x}}$, $\mathbf{e}_{\mathrm{y}}$ directions. In Eq.(21) $\delta$ is the Kronecker symbol and

$$
\phi(\alpha)=\mathcal{M}\left(\mathcal{M}(\alpha, L), L_{0}\right), \quad \xi(\alpha)=\mathcal{M}\left(\mathcal{I}(\alpha, L), L_{0}\right)
$$

are dependences illustrated in Fig. 16. The position of some dot including site $\alpha$ can be also written in terms of $\mathcal{M}$ and $\mathcal{I}$ functions:

$$
\mathbf{r}_{i(\alpha)}^{\mathrm{d}}=\mathcal{I}\left(\mathcal{M}(\alpha, L), L_{0}\right) \mathbf{e}_{\mathrm{x}}+\mathcal{I}\left(\mathcal{I}(\alpha, L), L_{0}\right) \mathbf{e}_{\mathrm{y}} .
$$

Here

$$
i(\alpha)=L_{\mathrm{d}} \mathcal{I}\left(\mathcal{I}(\alpha, L), L_{0}\right)+\mathcal{I}\left(\mathcal{M}(\alpha, L), L_{0}\right)
$$

is one-dimensional map associating site index $\alpha$ with dot index $i(\alpha) \in\left\{0,1,2, \ldots, L_{\mathrm{d}}^{2}-1\right\}$. 


\section{A.2 Integration scheme}

To derive explicit integration scheme with variable time steps $\kappa_{1} \Delta \tau$ and $\kappa_{2} \Delta \tau$ it is supposed that integral of spin angular frequency $\bar{\omega}_{\alpha}(\tau)$ is approximated by

$$
\int_{\tau}^{\tau+\Delta \tau} \omega_{\alpha}\left(\tau^{\prime}\right) \mathrm{d} \tau^{\prime}=\Delta \tau \bar{\omega}_{\alpha}(\tau)
$$

where

$$
\bar{\omega}_{\alpha}(\tau)=a_{0} \omega_{\alpha}(\tau)+a_{1} \omega_{\alpha}\left(\tau-\kappa_{1} \Delta \tau\right)+a_{2} \omega_{\alpha}\left(\tau-\kappa_{1} \Delta \tau-\kappa_{2} \Delta \tau\right)
$$

is $\tau$-local $\Delta \tau$ averaged $\omega_{\alpha}\left(\tau^{\prime}\right)$ expressed through coefficients

$$
\begin{aligned}
& a_{0}=\frac{2+6 \kappa_{1}^{2}+3 \kappa_{2}+6 \kappa_{1}\left(1+\kappa_{2}\right)}{6 \kappa_{1}\left(\kappa_{1}+\kappa_{2}\right)}, \\
& a_{1}=-\frac{2+3 \kappa_{1}+3 \kappa_{2}}{6 \kappa_{1} \kappa_{2}} \\
& a_{2}=\frac{2+3 \kappa_{1}}{6 \kappa_{2}\left(\kappa_{1}+\kappa_{2}\right)}
\end{aligned}
$$

derived by equating to zero the series of difference [lhs.Eq.(25) - rhs.Eq.(25)] considered up to the order $\mathcal{O}\left(\Delta \tau^{4}\right)$. The reason for use of non-constant prefactors $\kappa_{1,2} \sim 1$ is the opportunity to change adaptively integration step via the instantaneous spin rotation extreme

$$
\max _{\alpha=0, \ldots, L^{2}-1} \bar{\omega}_{\alpha}\left(\tau-\kappa_{1} \Delta \tau\right) .
$$

In the uniform case $\kappa_{1}=\kappa_{2}=1$, the quadrature is compatible with Adams-Bashworth 3th order integration formula. The initial two steps estimating $\omega_{\alpha}(\tau-\Delta \tau)$ and $\omega(\tau-2 \Delta \tau)$ can be performed using Euler scheme obtained for $a_{0}=1, a_{1}=a_{2}=0$. 


\section{References}

[1] G. Prinz, K. Hathaway (Eds.), Physics Today, AIP, New York, 1995, pp.24-63, special issue of this subject.

[2] M. Natali, A. Lebib, Y. Chen, I.L. Prejbeanu, K. Ounadjela, J.Appl.Phys. 91 (2002) 7041.

[3] S.H. Liou, R.F. Sabiryanov, J.Magn.Magn.Mater 226 (2001) 1270.

[4] T. Leineweber, H. Kronmüller, J.Magn.Magn.Mater 192 (1999) 575.

[5] E.Yu. Vadmedenko, A. Ghazali, J.C.S. Lévy, Phys.Rev.B 59 (1999) 3329.

[6] O. Iglesias, A. Labarta, J.Magn.Magn.Mater 221 (2000) 149.

[7] H. Kachkachi, M. Nogues, E. Tronc, D.A. Garanin, J.Magn.Magn.Mater 221 (2000) 158.

[8] S. Santamaria, H.T. Diep, J.Appl.Phys. 91 (2002) 6872.

[9] K.L. Metlov, phys.stat.sol.(a) 189 (2002) 1015.

[10] S. Haykin, "Neural Networks", by Prentice Hall, Inc. Simon and Schuster, New Jersey, 1999.

[11] D. Horváth, M. Gmitra, I. Vávra, J.Magn.Magn.Mater 231 (2001) 273.

[12] D. Horváth, M. Gmitra, J.Magn.Magn.Mater 256 (2003) 195.

[13] M.E. Schabes, H.N. Bertram, J.Appl.Phys. 64 (1988) 1347.

[14] H.V. Jones, R.W. Chantrell, J.Appl.Phys. 91 (2002) 8855.

[15] R. Kikuchi, J. Appl. Phys. 27 (1956) 1352.

[16] Yu.P. Kalmykov, S.V. Titov, Phys. Solid. State 42 (2000) 918.

[17] H.B. Braun, Phys. Rev. Lett. 71 (1993) 3557.

[18] M. Bauer, J. Fassbender, B. Hillebrands, Phys.Rev.B 61 (2000) 3410.

[19] D. Hinzke, U. Nowak, J. Magn. Magn. Mater. 221 (2000) 365.

[20] J.J. Miles, B.K. Middleton, J.Magn.Magn.Mater 95 (1991) 99.

[21] H.B. Braun, Phys.Rev.B 50 (1994) 16485.

[22] G.A. Carpenter, S. Grossberg, Applied Optics 26 (1987) 4919.

[23] V. Honavar, L. Uhr, "Artificial Intelligence and Neural Networks: Step Toward Principled Integration", Academica Press, Boston, 1994. 


\section{B Figure Captions}


Figure 1: The array snapshot including configuration with the resolution given by $N_{\mathrm{S}}=4=2 \times 2$. Obtained for parameters $D / J=0.1, L_{0}=20$ at characteristic time $\tau_{\text {ch }}=8.516$. The differences between coherent rotation at array corners and flower mode near to the array center are striking. The dashed lines inside magnified segments a), b) belong to bounds separating dots. Along these lines the exchange couplings are broken. 
a)

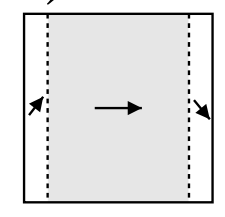

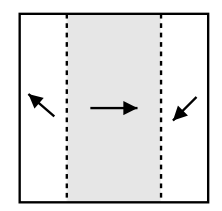

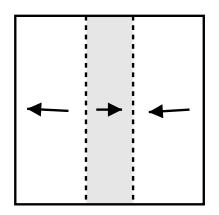

b)

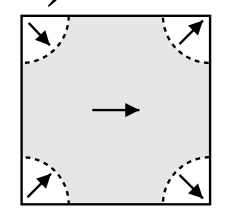

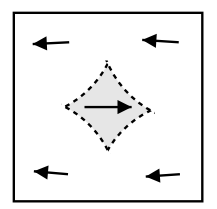

time.

Figure 2: Two schemes showing principal transients of nucleation, WAP formation and annihilation. The intra-dot open (straight) WAP (case a) occurring at earlier transients of remagnetization in eccentric dots. The center-symmetric WAP closed mode (case b) typical for late transients and dots positioned near the array center.

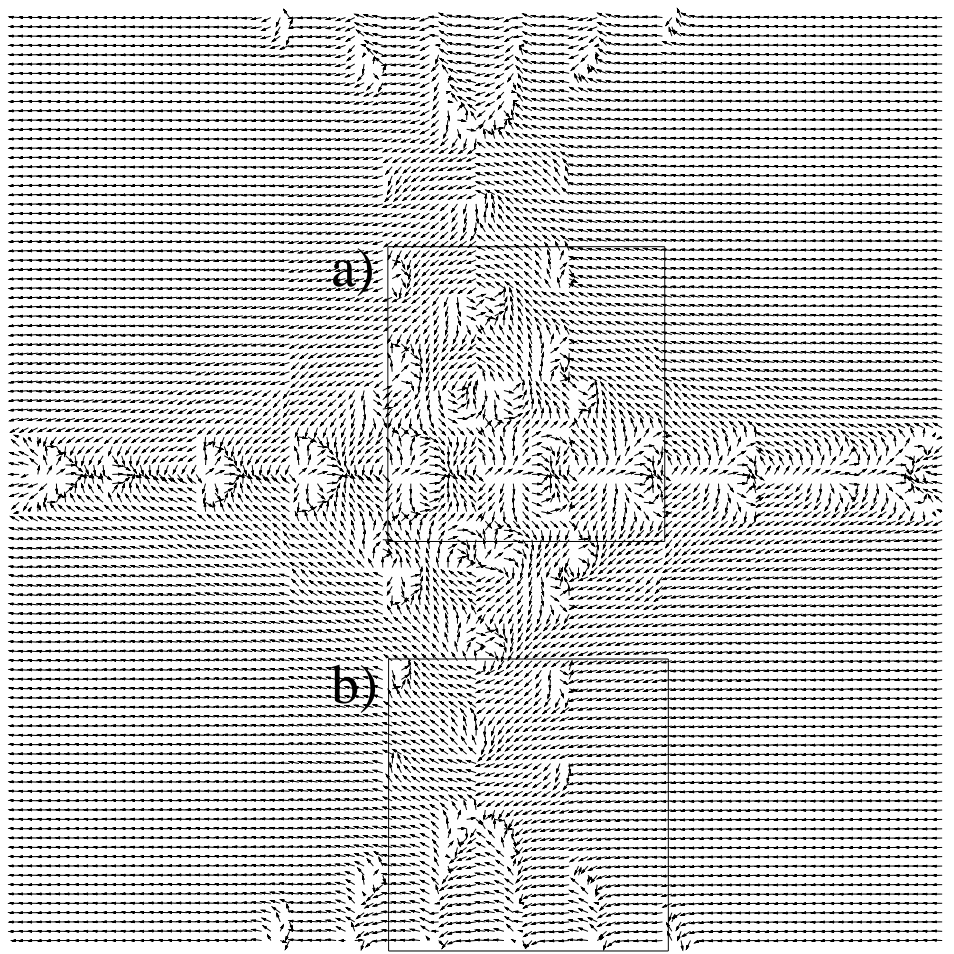

a)

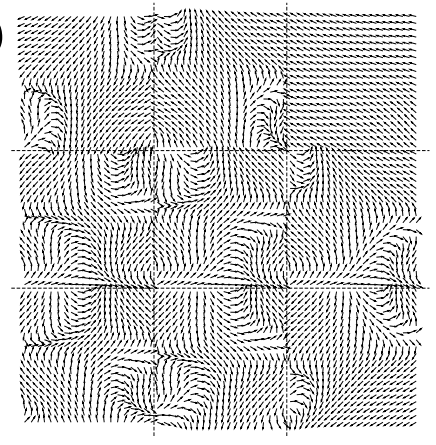

b)

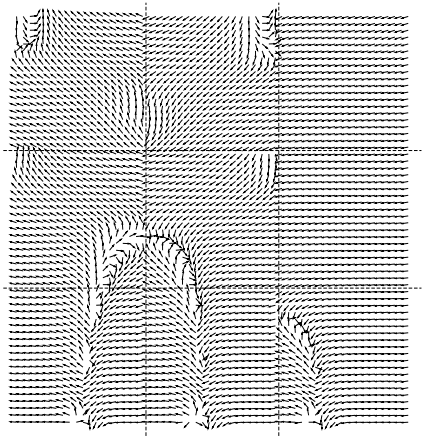

Figure 3: The array snapshot and its magnified details a), b) obtained for late stage $(\tau=11)$ of reversal (the dot labels and model parameters are identical as in the Fig. 1) nearly the time when the internal energy (free from Zeeman term) reaches its maximum. 


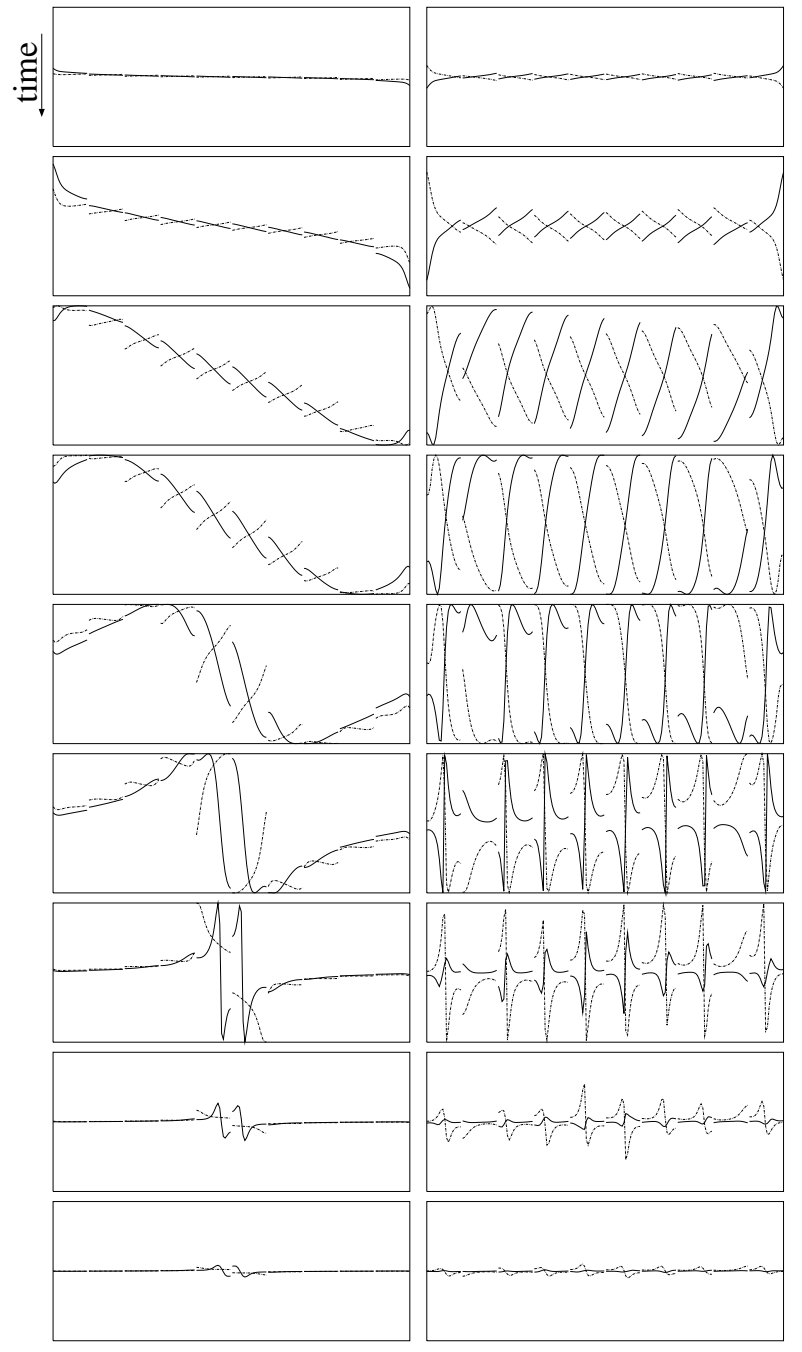

Figure 4: The reversal in the representation of one dimensional spin projections $\mathbf{S}_{l}(t) \cdot \mathbf{e}_{\mathrm{y}}$. The left hand side sequence of figures constructed for $22^{\text {nd }}$ stright line (solid line) and for $37^{\text {th }}$ line (dashed line) in the external field direction $\mathbf{e}_{\mathrm{x}}$. The right hand side sequence belongs to $97^{\text {th }}$ (solid line) and $102^{\text {nd }}$ line (dashed line) of the considered system $D / J=0.1, L_{0}=20$.

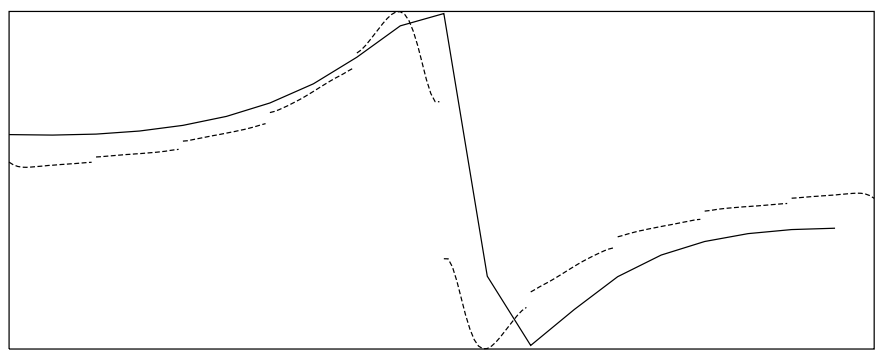

Figure 5: The snapshot illustrating similarity of nonlinear WAP at intra-dot $\left(L_{0}=20\right)$ and inter-dot $(L=200)$ spatial scales (at different times). The rescaling is performed for dot indexed by $i=55$ taken from $102^{\text {nd }}$ line of array [spin index changes within $\alpha \in\langle 20500,20519\rangle$ ] at time $\tau=13.5$ (depicted by solid line) and for $28^{\text {th }}$ spin line of array [where $\alpha \in\langle 5600,5799\rangle$ ] at $\tau=9.7$ (dashed line). The inter-dot and intra-dot configurations look similar when the spin coordinates are transformed using multiplicative factor $L_{0} / L$. 


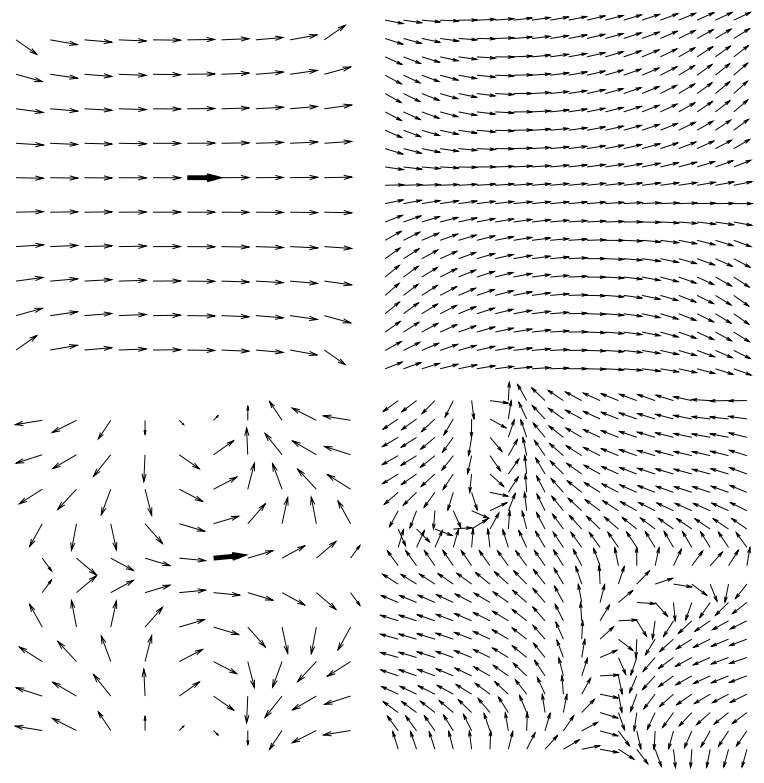

Figure 6: The spatio-temporal similarity of the magnetic configurations occurring at intra-dot and array scales. On the left hand side we plot overall moments of dots at different times $\tau=4.5$ (upper array flower) and $\tau=8.5$ (bottom array configuration). The detailed intra-dot configurations shown at the right hand side column corresponding to dot moments marked by the bold vector and fixed at the later times $\tau=8.5$ (upper intra-dot flower) $\tau=12.5$ (bottom three-domain intra-dot configuration) for $D / J=0.1, L_{0}=20, L=200$.

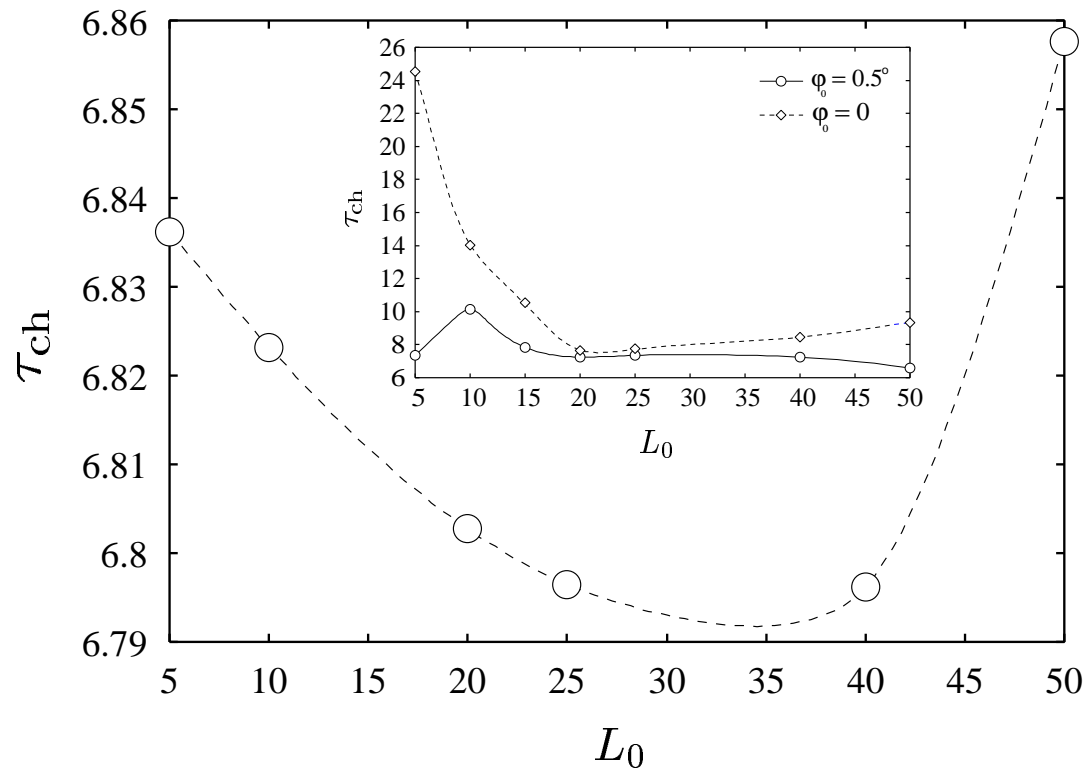

Figure 7: The $\tau_{\mathrm{ch}}\left(L_{0}\right)$ dependence calculated for $D / J=1, \mathbf{H} / J=-\mathbf{e}_{\mathrm{x}}$. The insert compares characteristic time for $\varphi_{0}>0$ and $\varphi_{0}=0$ configurations in isolated dot. The most remarkable are differences for small $L_{0}$. The lines are guidelines to eye. Only a weak dependence on hierarchical cut-off is observed. The test of $\tau_{\text {ch }}$ accuracy for a given size of array is performed for $L_{0}=5$ and $r_{\text {cut }} \in\left\{\sqrt{2} L_{0}, 2 \sqrt{2} L_{0}, 3 \sqrt{2} L_{0}\right\}$. The calculations confirm that changes in $\tau_{\text {ch }}$ does not exceed bound 0.004 . 

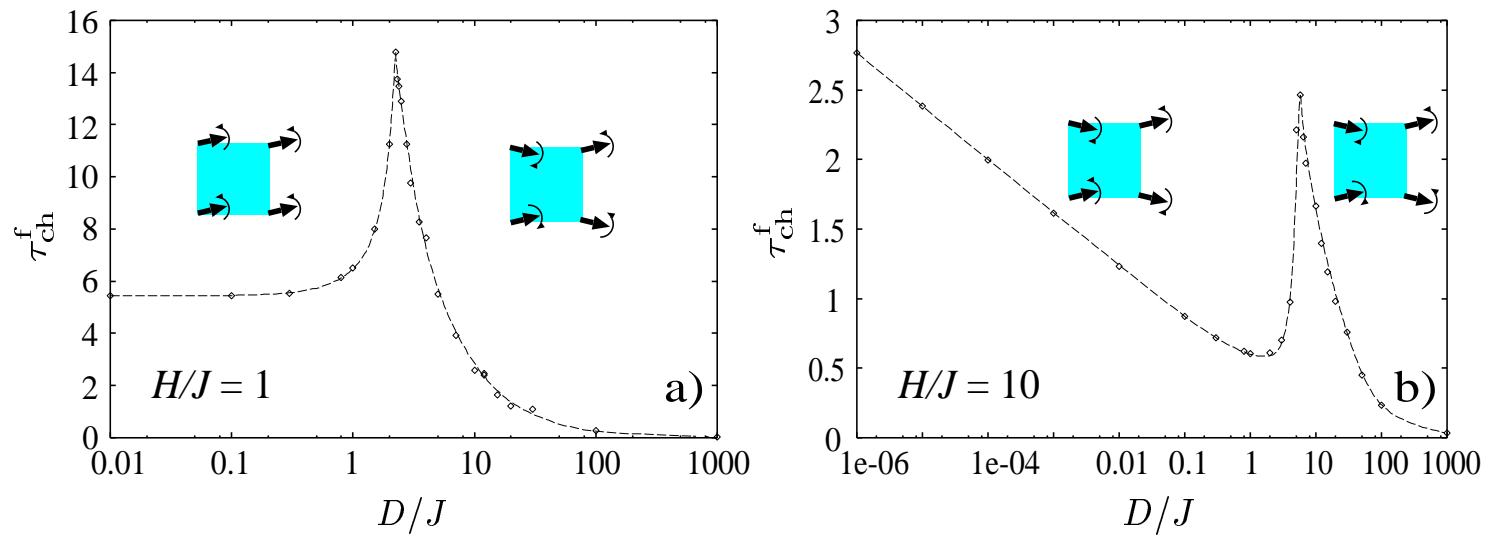

Figure 8: The $\tau_{\mathrm{ch}}(D / J)$ dependences obtained for different remagnetization (flower and vortex) modes of four-spin system simulated for $\varphi_{0}>0$ (left hand side) and for $\varphi_{0}=0$ (right hand side). The cusp corresponds to four-spin bifurcation point $D_{\mathrm{c}}^{\mathrm{f}}$.

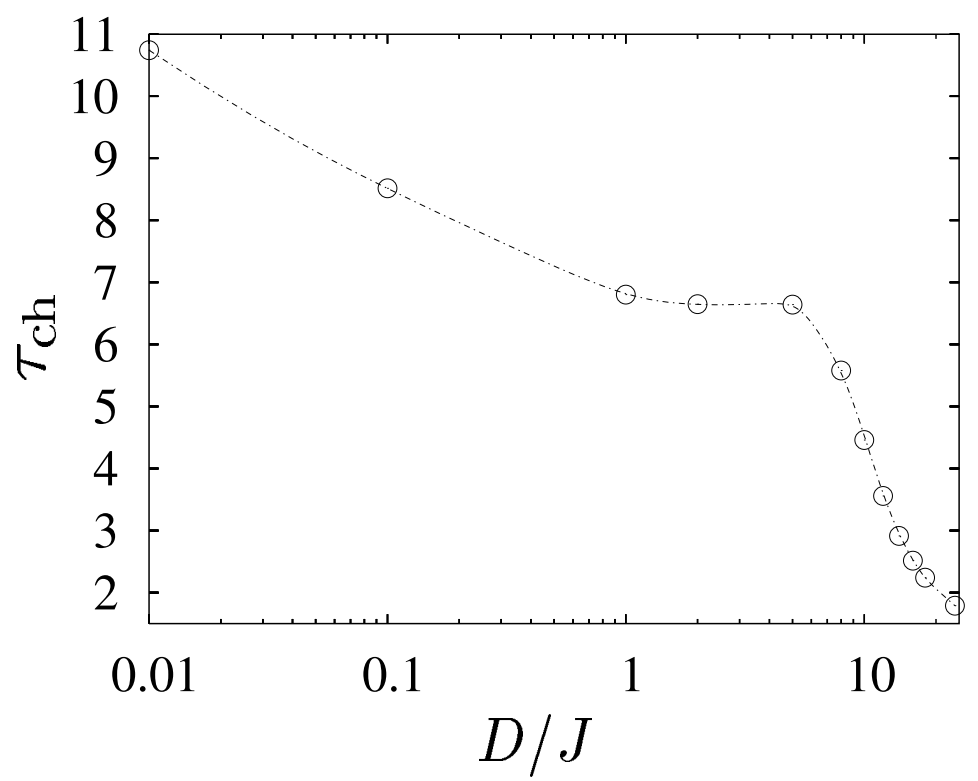

Figure 9: The plot of $\tau_{\mathrm{ch}}(D / J)$ dependence obtained for modulation $L_{0}=20$. The variation of $\tau_{\mathrm{ch}}(D / J)$ slope near $D_{\mathrm{c}}$ associated with change of reversal mode. 


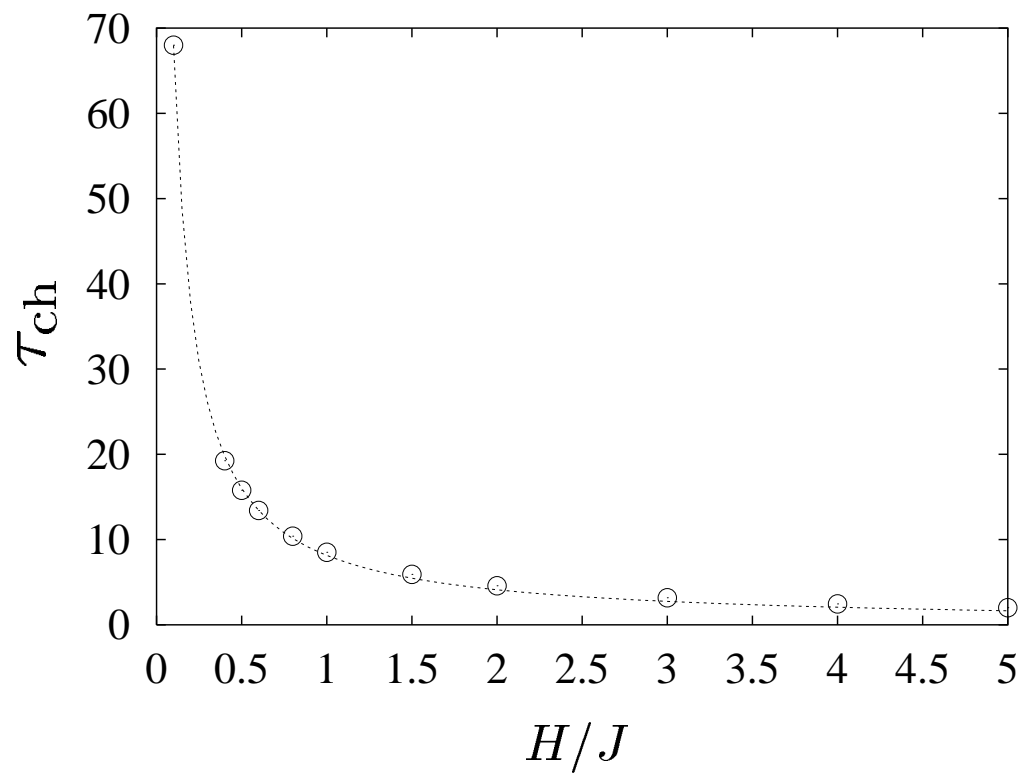

Figure 10: The plot of $\tau_{\mathrm{ch}}(H / J)$ dependence obtained for $L_{0}=20$ and $D / J=0.1$. Dashed line represents fit $\tau_{\text {ch }}=a(J / H)-b D\left(J / H^{2}\right)$ with parameters $a=8.302 \pm 0.122$ and $b=1.506 \pm 0.131$. The choice of fitting function stems from the asymptotic $H$-dependence derived for four-spin model in sect.3.2.2.

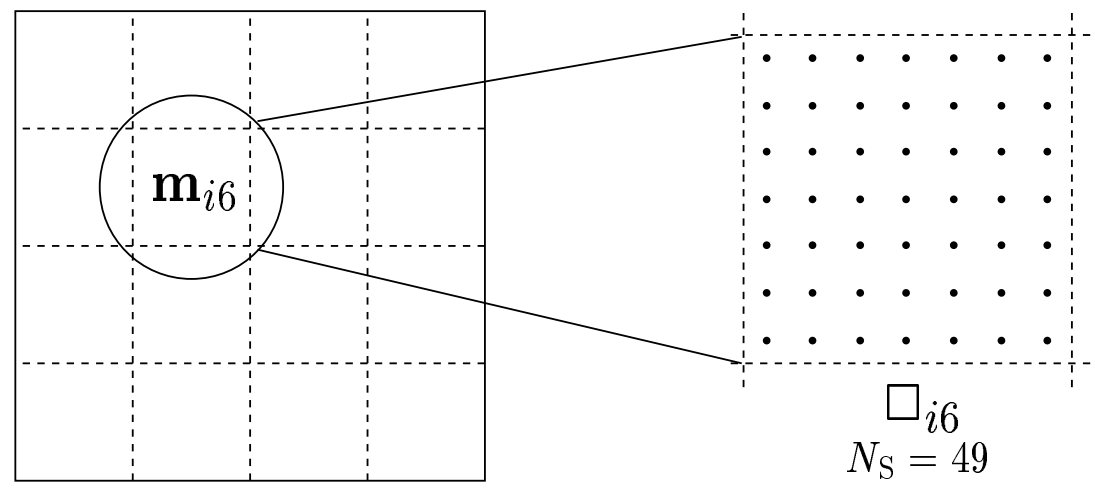

Figure 11: The schematic example of intra-dot segmentation. Figure showing sixth square segment labeled $\square_{i 6}$ belonging to $i$ th dot including $N_{\mathrm{S}}=49$ spins. 


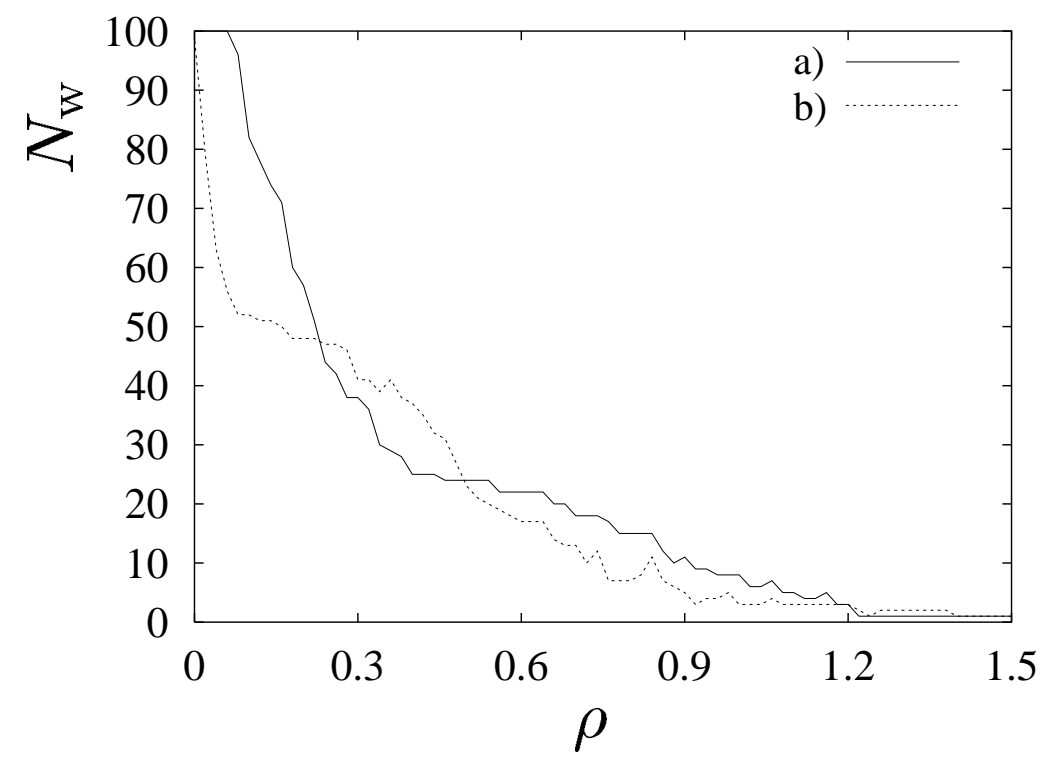

Figure 12: The pruning of ART neurons. The number of neurons $N_{\mathrm{w}}$ decreases with increasing vigilance parameter. The dependences obtained for $D / J=0.1$ and $L_{0}=20$. The intra-dot configuration is locally averaged over $N_{\mathrm{S}}=4$ spins [see Eq.(12)]. Case a) corresponds to configuration near to the switching time $(\tau=8.5)$. Case b) belongs to time when the internal energy (free from Zeeman term) is near its maximum $(\tau=11)$.

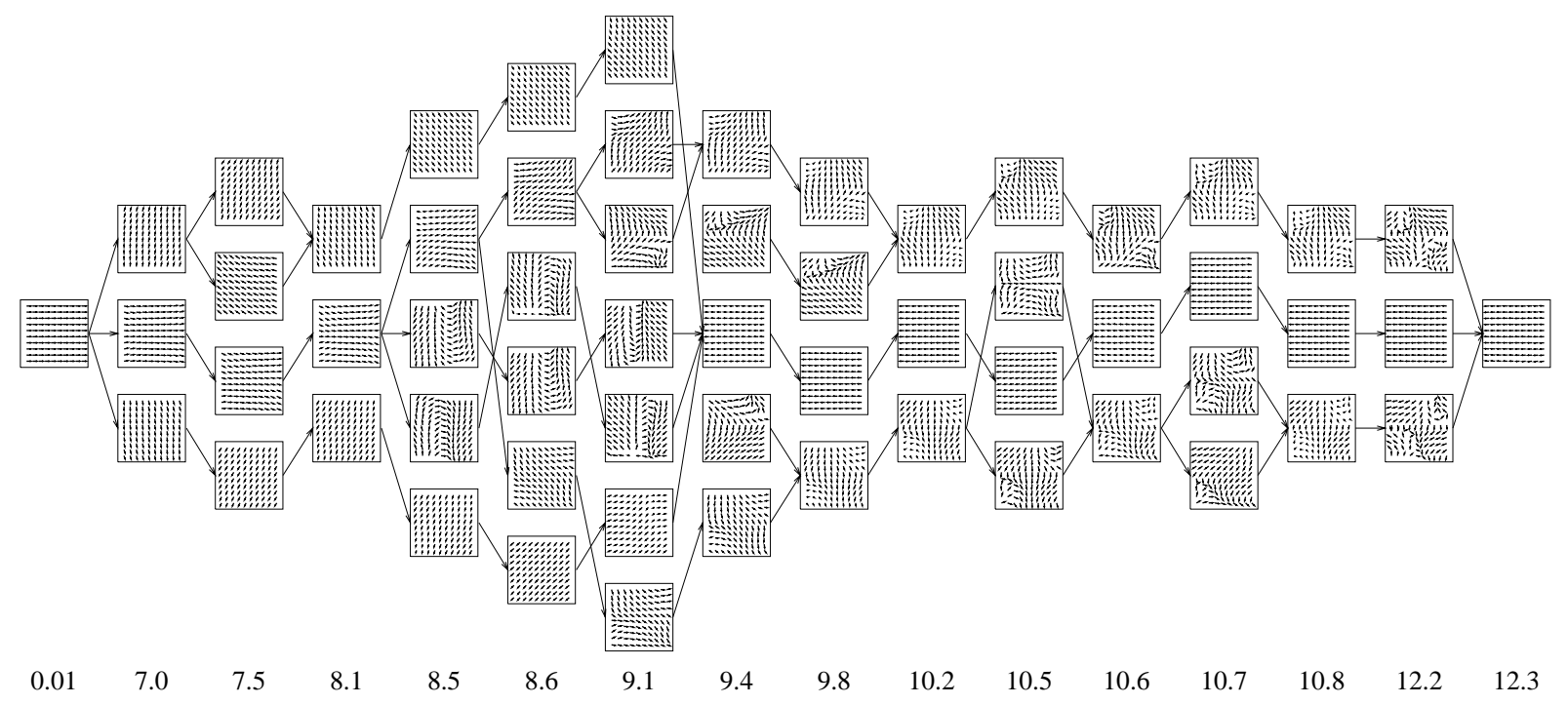

Figure 13: The ART network representation of the main intra-dot transients of array reversal for $D / J=0.1, L_{0}=20$. The configurations corresponding to different times obtained for $N_{\mathrm{S}}=4$ segment average [see Eq.(12)]. The arrows connect closest transient configurations (in the sense of the magnetic Euclidean distance). The extremal number of neurons occurring for $\tau=9.1$ roughly corresponds to the maximum of internal energy. 


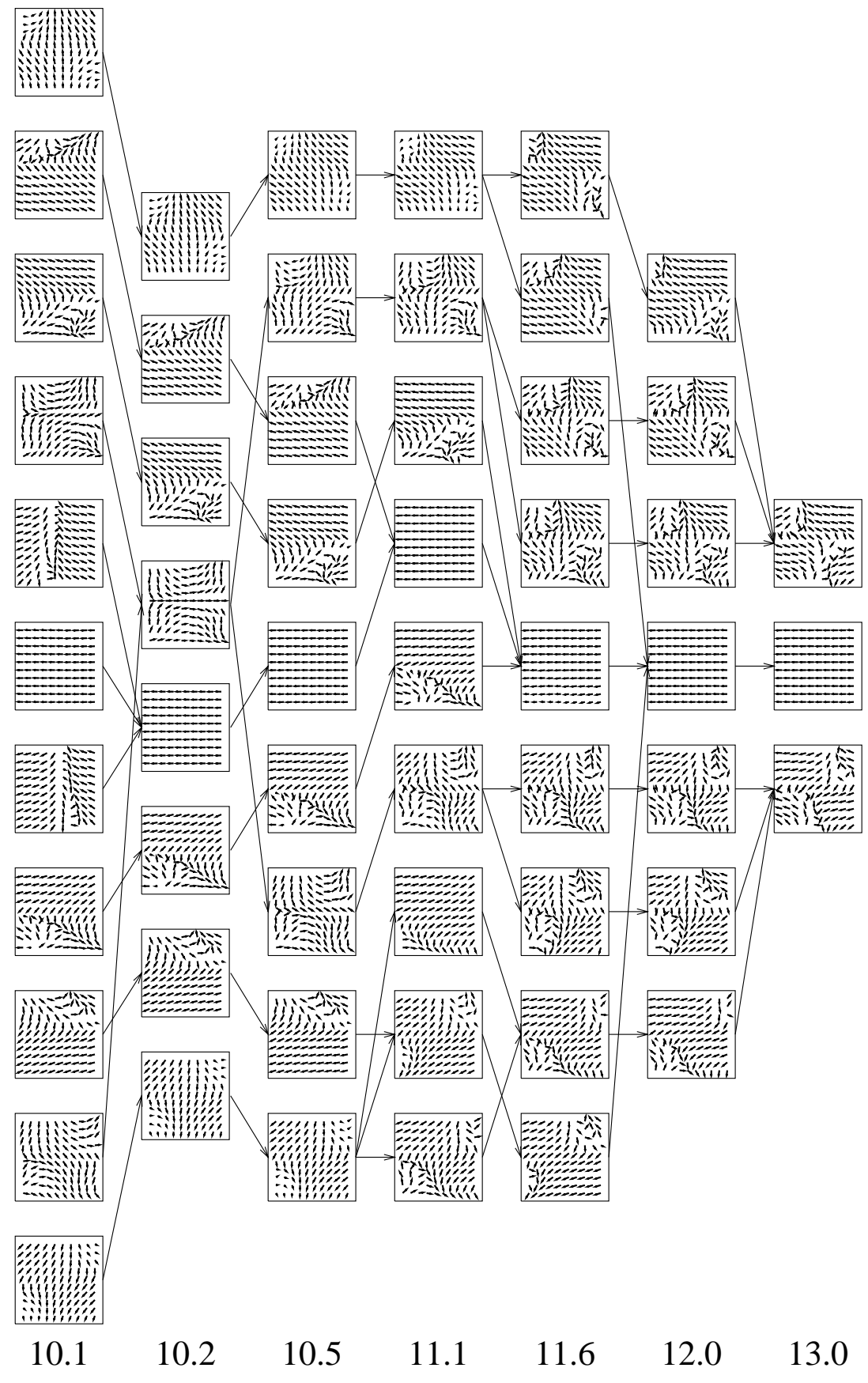

Figure 14: Figure showing ART trained with relatively small vigilance $\rho=0.8$. The network properly uncovers mechanism of WAP annihilation and nonlinear wave motion. 


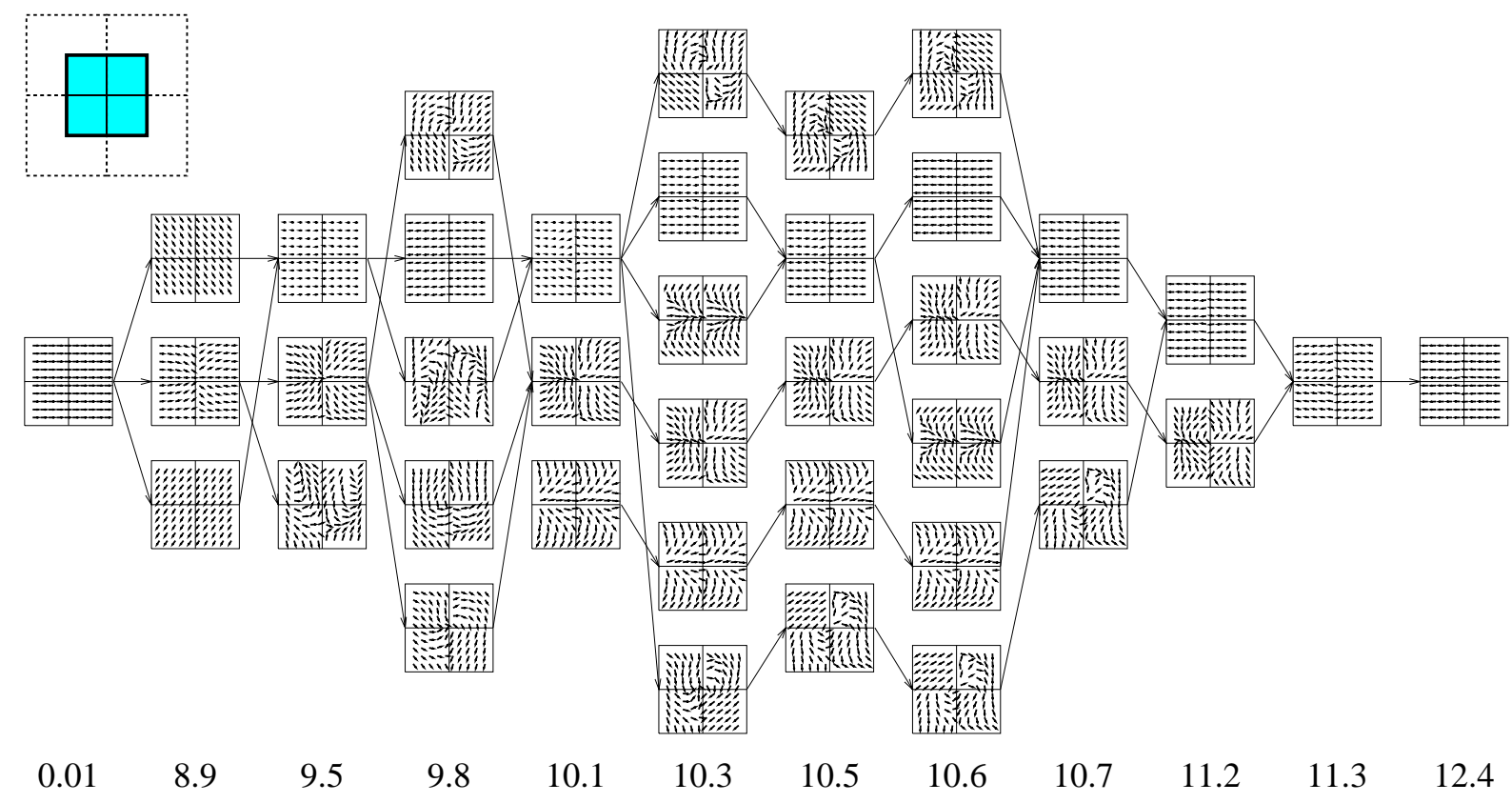

Figure 15: The ART revealing interfacial four-dot modes of magnetization reversal: inter-dot nonlinear wave pinning and formation of inter-dot vortices. Parameters: $D / J=0.1, \rho=1.22, N_{\mathrm{S}}=4, L_{0}=10$.

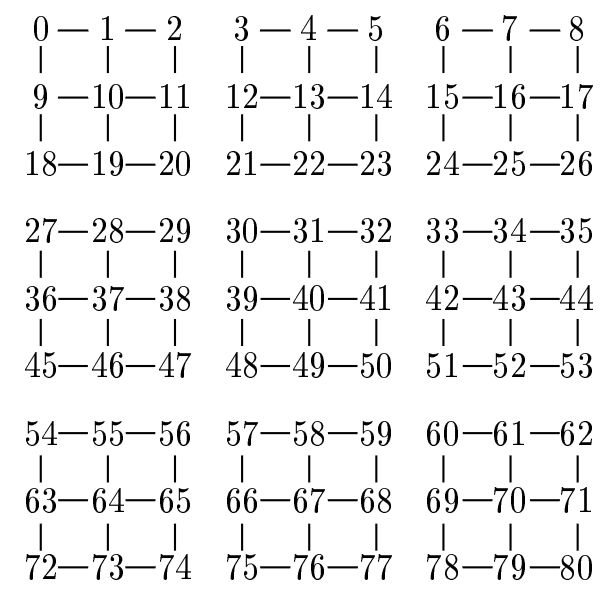

$\phi(\alpha) \quad \xi(\alpha)$

\begin{tabular}{|c|c|c|c|c|c|c|c|c|c|c|c|c|c|c|c|}
\hline 1 & 2 & 0 & 1 & 2 & 0 & 1 & 2 & 0 & & & & & 0 & & \\
\hline 1 & 2 & 0 & 1 & 2 & 0 & 1 & 2 & 1 & & & & & 1 & 1 & 1 \\
\hline 1 & 2 & 0 & 1 & 2 & 0 & 1 & 2 & 2 & & & 2 & $z$ & 2 & 2 & 2 \\
\hline 1 & 2 & 0 & 1 & 2 & 0 & 1 & 2 & 0 & 0 & 0 & 0 & 0 & 0 & 0 & 0 \\
\hline$\vdots$ & & $\vdots$ & $\vdots$ & $\vdots$ & $\vdots$ & $\vdots$ & $\vdots$ & $\vdots$ & & & $\vdots$ & & $\vdots$ & $\vdots$ & $\vdots$ \\
\hline 1 & & 0 & 1 & , & 0 & & 0 & 2 & & & 0 & 8 & 2 & 2 & \\
\hline
\end{tabular}

Figure 16: The illustration of one-dimensional site enumeration scheme $\left(L_{0}=3, L=9\right)$. The modulation of connectivity matrix $\epsilon_{\alpha \beta}$ with period $L_{0}=3$ in $\mathbf{e}_{\mathrm{x}}$ and $\mathbf{e}_{\mathrm{y}}$ perpendicular directions realized by functions $\phi(\alpha), \xi(\alpha)$ from Eq. $(21)$. 